\title{
Stimulus and response factors in mirror-image discrimination
}

\author{
JOSHUA STALLER and ROBERT SEKULER \\ Cresap Neuroscience Laboratory, Northwestern University, Evanston, Illinois 60201
}

\begin{abstract}
Two choice-reaction time studies assessed the influence of stimulus-response mapping, stimulus complexity, and stimulus alignment on adults' discrimination of mirror-image and nonmirror-image stimulus pairs. Half the subjects in Experiment 1 were instructed to treat nonmirror pairs as "same" and mirror pairs as "different"; the other half responded in the opposite manner. The first group responded more quickly to nonmirror pairs, while the second group responded more quickly to mirror pairs. This result, which held for horizontal stimuli (side by side) as well as for vertical stimuli (one above the other), confirms the importance of experiential factors in mirror-image "confusions." In Experiment 2, stimuli were drawn from a population of patterns whose complexity could be objectively defined. In general, the more complex the pattern, the slower the response and complexity seemed to influence the qualitative nature of pattern processing. In both experiments, subjects responded more quickly to horizontal stimuli than to vertical stimuli.
\end{abstract}

Humans of all ages have dịfficulty processing shapes that are mirror images of one another. A familiar manifestation of mirror-image confusion occurs when children learn to read. Typically, " $b$ " is confused with " $d$," or " $p$ " is confused with "q." Less frequently, " $n$ " is confused with " $u$," and " $m$ " with " $w$ " (Smith, 1928). Similar confusions of letters or letter-like shapes can be experimentally induced in normal adults (Krise, 1952; Rock \& Harris, 1967).

Many theorists have tried to explain the occurrence of mirror-image errors. Barroso and Braine (1974) have suggested that these theories can be classified into two major categories: structural and experiential. A structural theorist (e.g., Orton, 1937) might argue that mirror-image confusions result from competing contralateral hemispheric representations. In contrast, an experiential theorist (e.g., Gibson; 1969) would emphasize that, in the absence of learning and information feedback, an observer might fail to treat left-right orientation as a relevant dimension among shapes.

One standard test of mirror-image discrimination is illustrated in Figure 1 A. The subject is shown two stimuli, and he must decide if they are the "same" or "different." Pairs 1 and 2 (nonmirror-image pairs) have usually been called "same" because both

This work was supported by Grants NS-10094 and EY-00321 from the National Institutes of Health. The senior author was also supported by NIMH Fellowship MH-05499 during the preparation of this manuscript. The senior author is now at the Department of Psychology, Vanderbilt University, Nashville, Tennessee. We thank Robert Armstrong for helpful comments and advice. components open to the left (1) or both open to the right (2). Pairs 3 and 4 (mirror-image pairs) have been called "different" since in each case one component opens leftward and one opens rightward. Note that these stimuli have been classified according to a "left-right" criterion. When children judge these stimuli, they make more errors on mirror pairs 3 and 4 than on nonmirror pairs 1 and 2 (Robinson \& Higgins, 1967). When adults judge these stimuli they respond more slowly to mirror pairs 3 and 4 than to nonmirror pairs 1 and 2 (Corballis, Miller, \& Morgan, 1971). So the relative difficulty of mirror pairs is similar for adults and children.

At first, the correspondence between adult reaction times and children's errors seems to cast doubt on an experiential explanation of mirror con-

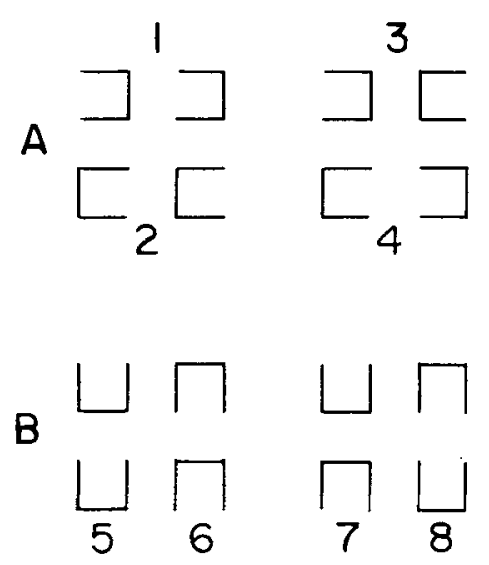

Figure 1. (A) Pair comparison stimuli $1-4$ in horizontal alignment. (B) Pair comparison stimuli 5-8 in vertical alignment. 
fusions. After all, it is unlikely that adults fail to attend to the left-right orientation of the stimuli. But a recent study by Staller and Sekuler (1976) suggests that the experiential approach is still viable. In that study, both adults and preschool children were tested. Half the subjects were instructed to use the traditional "left-right" response criterion-they treated pairs 1 and 2 as "same" and pairs 3 and 4 as "different." However, the remaining subjects used a new, "in-out" response criterion; they were instructed to treat pairs 3 and 4 as "same" (since both components open outward or both open inward) and pairs 1 and 2 as "different" (in each case one component opens inward and one opens outward).

The results were as follows. Subjects instructed to use the left-right criterion performed better on the nonmirror than on the mirror pairs, while subjects using the in-out criterion performed relatively better on the mirror pairs. The correspondence between adult reaction times and children's errors was preserved for both the in-out and the left-right groups. Thus, stimulus-response mapping plays a key role in determining the relative difficulty of mirror-image vs. nonmirror-image pairs for both children and adults.

The present experiments confirm and extend our previous findings. Though Staller and Sekuler (1976) used relatively complex dot stimuli in just one stimulus alignment condition, previous investigators often used simple line stimuli in a variety of alignment conditions (Cairns \& Steward, 1970; Huttenlocher, 1967; Sekuler \& Houlihan, 1968; Sekuler \& Rosenblith, 1964; Wolff, 1971). So, in Experiment 1, we asked adults to judge simple line stimuli in horizontal (Figure $1 \mathrm{~A}$ ) as well as vertical alignment (Figure 1B). Half the adults used an in-out criterion; half used a left-right criterion.' It was expected that the in-out group would respond relatively quickly to the mirror image pairs $(3,4,7,8)$, while the left-right group would respond more quickly to the nonmirror-image pairs $(1,2,5,6)$.

The manipulation of stimulus alignment also bears on the role of symmetry in pattern discrimination. As early as 1897 , Mach noted that horizontally aligned symmetric patterns seemed to be more salient than vertically aligned symmetric patterns, and he believed that the basis for this difference was the bilateral symmetry of the visual system. Recent experimental evidence tends to confirm Mach's insight (Deregowski, 1971; Hogben, Julesz, \& Ross, 1976; Julesz, 1971; Rock \& Leaman, 1963). Also, in addition to bilateral symmetry, Western man has considerable experience with horizontally aligned shapes (e.g., reading). On the basis of structural as well as experiential factors, then, one would predict that adults would respond more quickly to horizontally aligned stimuli.
A further consideration is the effect of stimulus complexity on mirror-image discrimination. We know that mirror confusions can occur with a wide variety of stimulus types, ranging from simple line stimuli (Rudel \& Teuber, 1963) to complex dot patterns (Staller \& Sekuler, 1976). But an explanation of mirror confusions at one complexity level may not hold at another complexity level. So Experiment 2 explores the effect of stimulus complexity using special patterns that lie on an objective continuum.

\section{EXPERIMENT 1}

The aim of the present study is bipartite: (1) to test further an experiential theory of mirror confusions by contrasting the in-out and left-right response criteria; and (2) to assess the role of the bilateral symmetry of the nervous system (and/or experience) in the discrimination of horizontal and vertical stimulus pairs.

\section{Method}

Subjects. Thirty-two college students (10 males, 22 females) were tested individually. All were paid volunteers.

Stimuli and Apparatus. Eight configurations of computergenerated patterns were presented under two alignment conditions (horizontal and vertical). Sample horizontal stimuli are shown in Figure 1A (pairs 1-4) and sample vertical stimuli are shown in Figure 1B (pairs 5-8).

Stimulus pairs were presented by a computer on a cathode ray display with phosphor similar to P1. Each component in a horjzontal pair consisted of three straight-line segments (one vertical, two horizontal) arranged in a horseshoe shape. A line segment was composed of 50 discrete points presented on the oscilloscope display; the high density of the points made the line segment appear continuous. Each component in a pair subtended approximately $1.4^{\circ} \times 1.4^{\circ}$, with the center of each displaced about $1.4^{\circ}$ from a central fixation point. The luminance of each component was approximately $1.71 \mathrm{~cd} / \mathrm{m}^{2}$ and the background luminance was $.06 \mathrm{~cd} / \mathrm{m}^{2}$. A high refresh rate $(99 \mathrm{~Hz})$ insured that the pair components appeared to be presented simultaneously and without flicker.

Procedure. The subjects were assigned randomly to one of two groups. Group 1 (left-right) was instructed to respond "same" to pairs $1,2,5$, and 6 , and "different" to pairs $3,4,7$, and 8 . In horizontal alignment, pairs 1 and 2 were considered "same" because both components open left or both open right. Pairs 3 and 4 were "different" because in each case one component opens left and one opens right. In vertical alignment, pairs 5 and 6 were considered "same" because both components open up or both open down. Pairs 7 and 8 were "different" because one component opens up and one opens down.

Group 2 (in-out) was instructed to respond "same" to pairs 3, 4,7 , and 8 , and "different" to pairs $1,2,5$, and 6 . Pairs 3, 4, 7 , and 8 were considered "same" because in each case both components open inward or both open outward. Pairs 1, 2, 5, and 6 were "different" because in each pair one component opens inward and one opens outward (the horizontal pairs, 1-4, were judged in terms of an imaginary vertical midline between pair components, while the vertical pairs, 5-8, were judged in terms of an imaginary horizontal midline between pair components). ${ }^{2}$

Each subject was seated in a darkened booth at a distance of $65 \mathrm{~cm}$ from the display. The nonpreferred eye was covered and 
the subject rested his head on a chinrest. Half the subjects in each group were first shown the patterns in vertical alignment (5-8) and then in horizontal alignment (1-4). The remaining subjects saw the horizontal patterns first. Within each order of alignment condition, two subjects pressed a lever up to signal "different" and down to signal "same," two responded up for "same" and down for "different," two responded left for "same" and right for "different," and two responded left for "different" and right for "same."

To indicate the start of a trial and provide a fixation point, an " $\mathrm{X}$ " appeared in the center of the display for $1 \mathrm{sec}$. Upon offset of the " $X$," one of the eight stimulus types was presented for $100 \mathrm{msec}$. Reaction time was defined as the interval from the of fset of the stimulus pattern to the subject's response. The intertrial interval was $4.5 \mathrm{sec}$.

Testing required one $45-\mathrm{min}$ session per subject. A session consisted of four blocks of test trials, 80 trials per block, with a short break between blocks. The subjects were given 20 practice trials (in the condition appropriate for the first test block). Within a trial block, the subjects made 20 correct responses to each pattern type. Whenever the subject made an error, the stimulus appearing on the error trial was randomly assigned a new position in the presentation queue and shown again at the appropriate time. Under the constraints described above, the pattern appearing on any particular trial was randomly determined.

\section{Results}

The overall error rate was small (mean $=1.6 \%$ ) with no substantial differences between groups or between stimulus alignment conditions. An analysis of variance was performed on reaction times for correct responses (switch throw assignment and order of alignment conditions were ignored in the analysis). With respect to reaction times, one prediction of interest is that both groups would be quicker on "same" than on "different" judgments. Indeed, overall, the left-right group was faster on nonmirror patterns (mean $=596 \mathrm{msec}$ ) than on mirror patterns (mean $=652 \mathrm{msec}$ ), while the in-out group was faster on mirror patterns (mean $=560 \mathrm{msec}$ ) than on nonmirror patterns (mean $=578 \mathrm{msec}$ ). Consistent with this view, the analysis of variance revealed a significant Group by Pattern Type interation, $F(7,210)=4.96, p<.001$.

A Newman-Keuls analysis determined which of the eight pairs contributed to the Group by Pattern Type interaction. The mean for each pattern type is plotted in Figure 2. For the horizontally aligned pairs, $1-4$, the subjects responded significantly faster to mirror pair 4 when using an in-out criterion than when using a left-right criterion, $\mathrm{p}<.05$. The subjects also appeared to respond faster to mirror pair 3 when using an in-out criterion, but this difference did not reach significance, $.05<\mathrm{p}<.10$. For vertically aligned pairs $5-8$, a similar pattern of results is observed; subjects responded significantly more quickly to mirror pairs 7 and 8 when using an in-out criterion than when using a left-right criterion, $\mathrm{p}<.05$. No other comparisons within each alignment condition reached significance. These results indicate that the Group by Pattern Type interaction was mediated largely by performance on mirror pairs; the in-out group tended to be faster than the left-right group on mirror pairs $3,4,7$, and 8 .

Possible performance differences between horizontal and vertical stimuli are also of interest in this experiment. Inspection of Figure 2 suggests that subjects responded more quickly to horizontally aligned stimuli. Indeed, the mean overall response time for horizontal stimuli was $577 \mathrm{msec}$, with a corresponding value for vertical stimuli of $616 \mathrm{msec}$. The analysis of variance term which bears most directly on the horizontal-vertical comparison is the main effect of pattern type. This effect was significant, $F(7,210)=6.11, \mathrm{p}<.001$. A NewmanKeuls analysis indicated that subjects responded more quickly to pair 2 than to pairs 5-8, more quickly to pairs 1 and 4 than to pairs 7 and 8 , and more quickly to pair 3 than to pair 7 , all $\mathrm{p}<.05$. In each case, the horizontally aligned pairs were responded to more quickly.

No other effects of the analysis of variance were significant, $\mathrm{p}>.1$.

This experiment supports previous findings. The left-right group tended to respond more quickly to nonmirror patterns, while the in-out group tended to respond more quickly to mirror patterns. The fact that this pattern of results was observed for both horizontally and vertically aligned simple line stimuli increases the generality of the Staller and Sekuler (1976) findings. Also, the faster responses to horizontal stimuli support the importance of experience and/or bilateral symmetry in mirror-image discrimination.

\section{EXPERIMENT 2}

This study examines the effect of stimulus complexity on mirror-image discrimination, using patterns that lie on an objective continuum of randomness. We assume that subjective complexity is a monotonic function of the degree of randomness (i.e., the degree of internal constraint; Snodgrass, 1971).

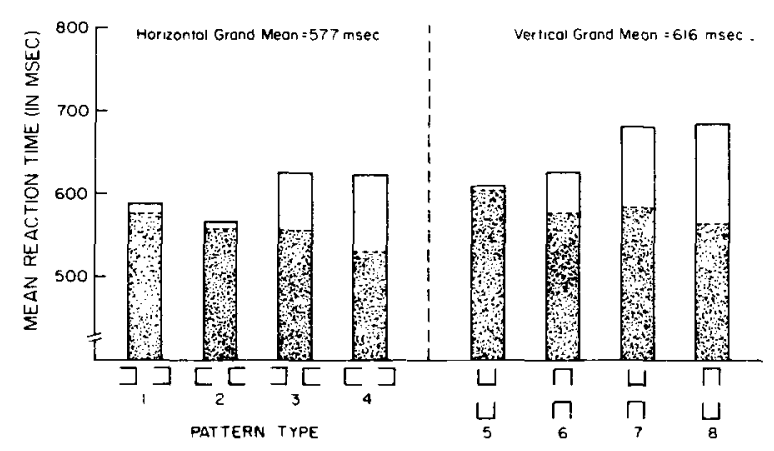

Figure 2. Mean response times (Experiment 1) for Stimuli 1-8 under left-right (unspeckled) and in-out (speckled) triteria. Each mean is based on 640 trials. 


\section{Method}

Subjects. Eight college students ( 2 males, 6 females) were tested individually. Four had served in Experiment 1. All were paid volunteers.

Stimuli and Apparatus. Eight different computer-generated patterns were presented under two conditions of alignment and five levels of complexity. ${ }^{3}$ Levels of complexity ranged from simple (Level 1) to complex (Level 5), as shown in Figure 3. Complexity Levels $1-5$ were combined factorially with each of the pattern types shown in Figures 1A and 1B. Simple stimuli (Level 1) corresponded exactly to those stimuli used in Experiment 1.

The size and separation of pattern components, as well as their luminance, refresh rate, and method of presentation, were the same as in Experiment 1.

Procedure. The procedure was the same as in Experiment 1, with the following exceptions. In Experiment 2, all subjects used a left-right criterion. On each trial, the stimulus pattern was plotted on the oscilloscope display until the subject made a response. Reaction time was defined as the interval from the onset of the stimulus pattern to the onset of the subject's response. Any response latency longer than $3 \mathrm{sec}$ was treated as an error. Testing required three 45-min sessions for each subject. The first session consisted of four blocks of test trials, 80 trials per block, with a short break between blocks. The second and third sessions consisted of three blocks of test trials, 80 trials per block, with a short break between blocks. Within a trial block, the subjects made 20 correct responses to each pattern type (four responses at each of the five complexity levels).

\section{Results}

The overall error rate was small (approximately $2 \%$ ) with no substantial difference between the two alignment conditions. There were, however, considerably more errors on Complexity Level 5 than on any of the other complexity levels.

The mean correct reaction times for each pattern type at each level of complexity are plotted in Figure 4 (horizontal pairs 1-4) and Figure 5 (vertical pairs 5-8). Each mean in Figures 4 and 5 is based on 160 trials. In general, response times increased with stimulus complexity: mean times for complexity Levels $1-5$ were $716,745,818,866$, and $1,042 \mathrm{msec}$, respectively.

We analyzed the variance from all the correct reaction times into components associated with complexity and pattern type. Each of these main effects was statistically significant, $F(4,28)=42.06, F(7,49)$ $=5.51$, respectively, $\mathrm{p}<.01$. In addition, we used the coefficients shown in Table 1 to decompose the effect of complexity into four orthogonal components. For each of these components, $\mathrm{df}=1,7$. First, over all five levels of complexity, the linear trend was significant, $F=44.63, p<.01$. Second, the data from the fifth level of complexity departed significantly from the linear trend established over the first four levels, $F=70.80, p<.01 .^{4}$ Neither quadratic nor cubic trends over the first four complexity levels were significant, $F=2.34$ and 2.07 , respectively, $p>.10$. Figures 4 and 5 show the pattern of components: a linear trend over the first four levels of complexity coupled with a departure from linearity introduced by the fifth level. The step
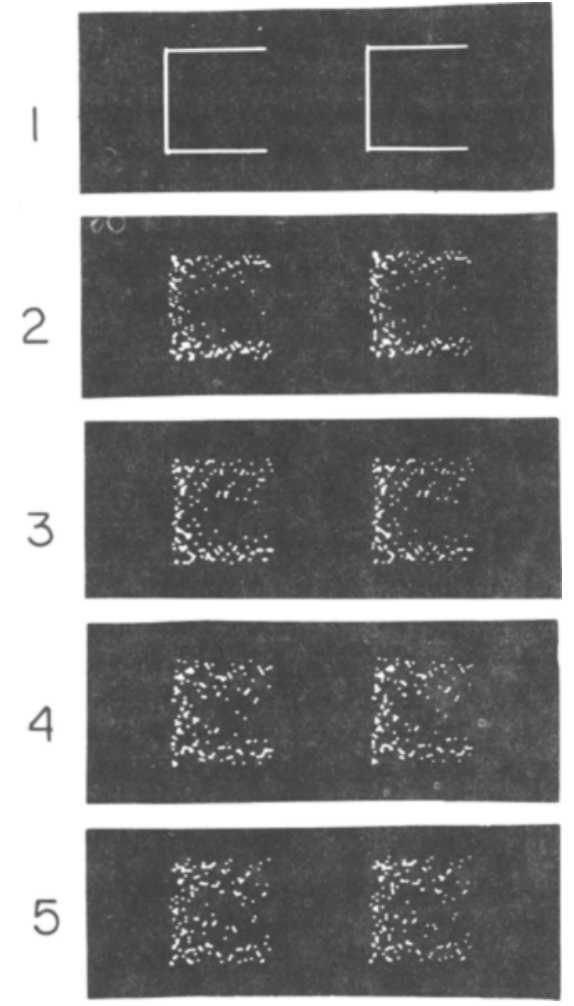

Figure 3. Computer-generated patterns at complexity Levels 1-5 (0-, 20-, 40-, 60-, and 80-step-long random walks, respectively). Each step was $2.5 \mathrm{~min}$ visual angle wide.

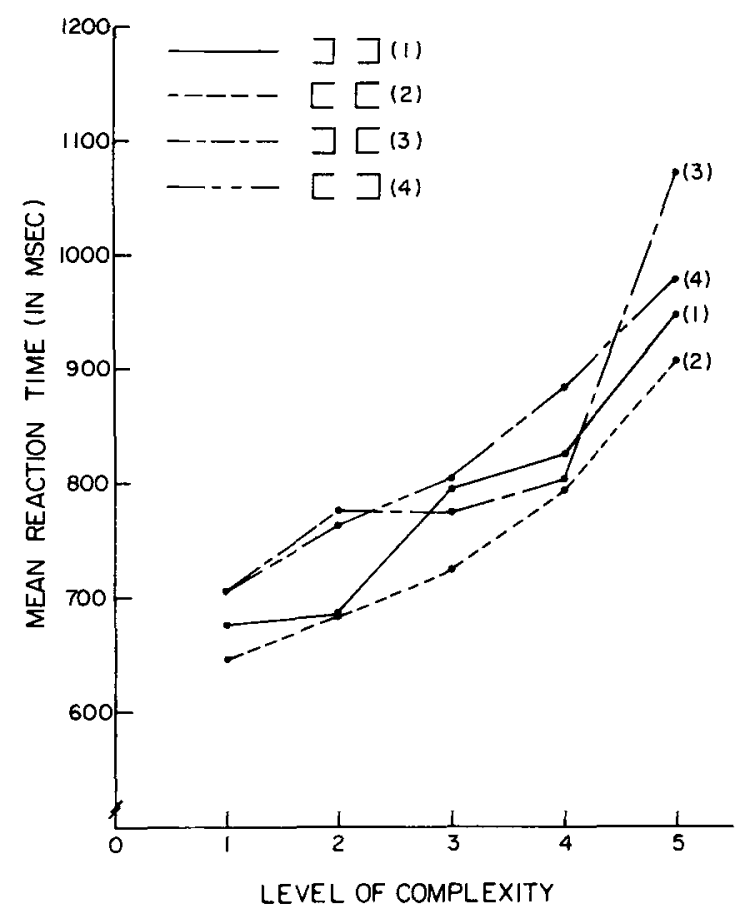

Figure 4. Mean response times (Experiment 2) for Stimuli 1-4 at complexity Levels 1-5. Each mean is based on 160 trials. 


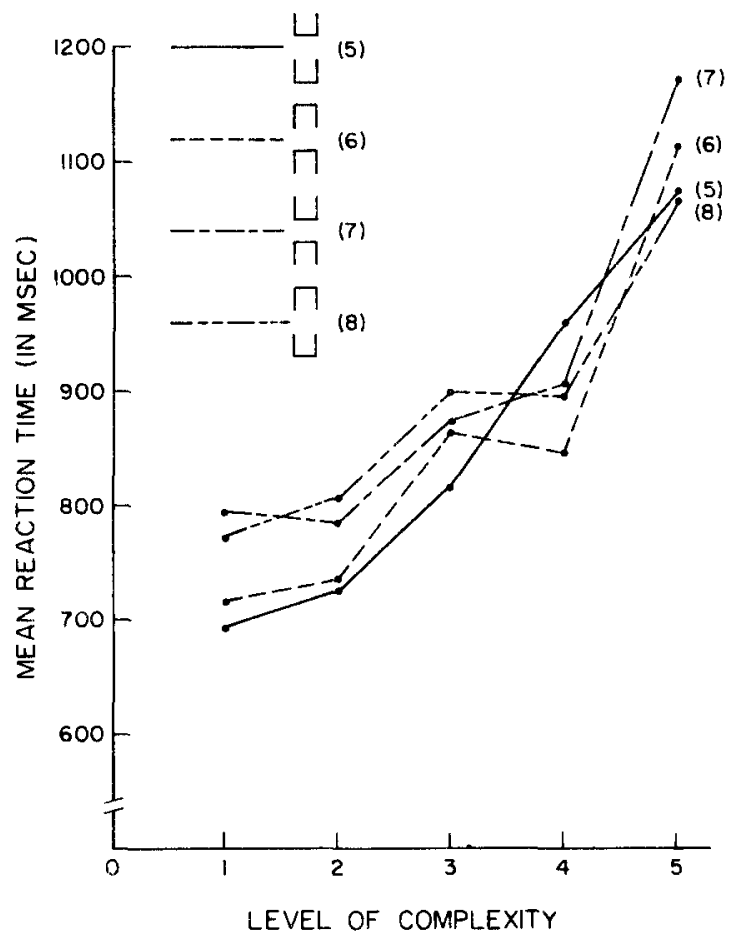

Figure 5. Mean response times (Experiment 2) for Stimuli 5-8 at complexity Levels 1-5. Each mean is based on 160 trials.

between complexity Levels 4 and 5 was larger than the subjective step between any of the other levels.

In general, then, increasing complexity produced slower response times. But, in addition, complexity exerted a qualitative effect on the processing of stimuli. A small, but significant, interaction between pattern type and complexity demonstrates this point, $F(28,196)=1.79, p<.05$. This effect is treated in more detail in the Discussion.

The mean response time for horizontal stimuli was $797 \mathrm{msec}$, while the corresponding value for vertical stimuli was $877 \mathrm{msec}$. The analysis of variance term which bears most directly on the horizontal-vertical comparison is the main effect of pattern type, which was significant, $F(7,49)=5.51, \mathrm{p}<.001$. A Newman-Keuls analysis showed that subjects responded more quickly to pair 2 than to pairs 5,6 , 7 , and 8 . Also, responses to pair 1 were quicker than responses to pairs 7 and $8, p<.05$. No other comparisons were significant. Subjects tended to

Table 1

Coefficient Vectors Used to Decompose Effect of Complexity

\begin{tabular}{lrrrrr}
\hline \multicolumn{1}{c}{ Term } & \multicolumn{5}{c}{} \\
\hline Linear & -2 & -1 & 0 & 1 & 2 \\
Nonlinear & -1 & 0 & 1 & 2 & -2 \\
Quadratic & 1 & -1 & -1 & 1 & 0 \\
Cubic & -1 & 3 & -3 & 1 & 0 \\
\hline
\end{tabular}

respond more quickly to stimuli in horizontal alignment.

To summarize: (a) the more complex the pattern, the slower the response; (b) the effects of stimulus complexity and pattern type were interactive rather than additive; and (c) as in the first experiment, subjects tended to respond more quickly to stimuli in horizontal than in vertical alignment.

\section{DISCUSSION}

Experiment 1 confirms the finding that the rapidity of adult judgments of pair comparison stimuli is determined to a significant extent by the response labels attached to mirror and nonmirror stimuli (Staller \& Sekuler, 1976). Stimulus pairs labeled "same" are judged more quickly regardless of whether these pairs are mirror stimuli or not; and this has been found for two stimulus alignment conditions (Experiment 1) as well as for two levels of stimulus complexity (Experiment 1; Staller \& Sekuler, 1976). Recently, Corballis and Roldan $(1974,1975)$ reported corroborative evidence of the influence of stimulusresponse mapping on adult reaction times to pair comparison stimuli. In addition, Staller and Sekuler (1976) have outlined possible linguistic factors and certain models of choice reaction time which seem to account for the overall pattern of results in adults.

The finding that subjects are sometimes superior on mirror stimuli strengthens an experiential theory of mirror confusions. It suggests that mirror stimuli cause special difficulty, not because of a tendency to perceive or remember them as identical, but because they are identical. All four pair types in Figure $1 \mathrm{~A}$ can be called "same"; therefore, partitioning these pairs into "same" and "different" is arbitrary. Since mirror stimuli are the same, problems arise when adults (or children) are forced to call them "different."

Experiment 2 focused on the effects of stimulus complexity. The results suggested that stimulus complexity has a qualitative effect on pattern processing, as indicated by a significant Pattern Type by Complexity interaction. We explore now the nature of this effect, confining our discussion to the Linear by Pattern Type interaction for complexity Levels 1-4. Visual inspection of Figures 4 and 5 suggests that two pair types contributed to the interaction-pairs 3 and 5. Pair 3 (see Figure 4) was responded to most slowly at Level 1 , though at Level 4 it was responded to more quickly than pairs 1 and 4 . Conversely, pair 5 (see Figure 5) elicited the fastest responses at Level 1 but the slowest responses at Level 4. Thus, over Levels $1-4$, response times to pair type 3 were relatively unchanged by increasing complexity, but response times to pair type 5 seemed particularly sensitive to increasing complexity. 
Hence, statements about the relative difficulty of judging different pair types must be conditional upon the complexity levels at which such comparisons are made. It is unclear, however, whether changes in complexity actually change the nature of pattern processing, or alternatively, whether the configuration of some stimuli makes them relatively immune to the effects of increasing complexity. Consider the latter alternative. Responses to pair type 3 might be less vulnerable to increasing complexity because dot density remains high near the center of the subject's gaze. Thus, the distinctive boundary features of pair 3 fall on or near the fovea and may therefore be relatively easy to evaluate as complexity increases.

A final consideration is the finding that subjects responded more quickly to stimuli in horizontal than in vertical alignment. This effect was recently reported by Corballis and Roldan (1975), who suggest that vertically aligned figures must be mentally rotated (to horizontal) before they can be mapped onto a hypothetical symmetry-detecting template in the brain. In support of the mental rotation hypothesis, horizontal stimuli were processed most quickly, followed by oblique pairs, followed by vertically aligned pairs. Thus the relative slowness of responses to vertical stimuli might be due to the extra time needed to rotate these stimuli in the mind's eye (e.g., Shepard \& Metzler, 1971). While the mental rotation hypothesis is an attractive one, other interpretations of the horizontal-vertical effect are possible. One alternative is that subjects have developed more efficient processing strategies for stimuli in horizontal alignment, presumably because they have considerable practice reading horizontally aligned text-and it is not uncommon to find ourselves reading obliquely oriented text from time to time.

\section{REFERENCES}

BarRoso, F., \& Braine, L. 'Mirror-image' errors without mirrorimage stimuli. Joumal of Experimental Child Psychology, 1974. 18. $213-225$.

Cairns. N..\& Stew ard, M. Young children's orientation of letters as a function of axis of symmetry and stimulus alignment. Child Development, 1970, 41. 993-1002.

Corballis, M., Miller, A., \& Morgan, M. The role of left-right orientation in interhemispheric matching of visual information. Perception \& Psychophysics, 1971, 10, 385-388.

Corballis, M., \& Roldan, C. On the perception of symmetrical and repeated patterns. Perception \& Psychophysics, 1974. 16. 136-142.

Corballis, M., \& Roldan, C. Detection of symmetry as a function of angular orientation. Journal of Experimental Psychology: Human Perception and Performance, 1975, 1, 221-230.

DeregowsKI. J. Symmetry, gestalt, and information theory. Quarterly Journal of Experimental Psychology, 1971, 23, 381-385.

Gibson, E. Principles of perceptual learning and development. New York: Appleton-Century-Crofts, 1969.

Hogben. J., Julesz. B., \& Ross. J. Short-term memory for symmetry. Vision Research, 1976, 16, 861-866.
Huttenlocher, J. Discrimination of figure orientation: Effects of relative position. Journal of Comparative and Physiological Psychology, 1967, 63. 359-361.

Julesz, B. Foundations of cyclopean perception. Chicago; University of Chicago Press, 1971.

KRISE, M. An experimental investigation of theories of reversals in reading. Journal of Educational Psychology, 1952, 43, 408-422.

MaCH. E. Analysis of the sensations. Chicago: Open Court, 1897. ORTON. S. Reading, writing and speech problems in children. London: Chapman and Hall, 1937.

Robinson, J., \& Higgins, K. The young child's ability to see a difference between mirror-image forms. Perceptual and Motor Skills, 1967. 25, 893-897.

Rock, I., \& Harris, C. Vision and touch. Scientific American, 1967. 216, 96-107.

Rock, I., \& LEAman, R. An experimental analysis of visual symmetry. Acta Psychologica, 1963, 21, 171-183.

Rudel, R., \& TeUber, H.-L. Discrimination of direction of line in children. Journal of Comparative and Physiological Psychology, 1963. 56, 892-898.

SeKuler, R., \& Houlihan, K. Discrimination of mirror images: Choice time analysis of human adult performance. Quarterly Journal of Experimental Psychology, 1968, 20, 204-207.

SEKUler. R. \& \& Rosenblith, J. Discrimination of direction of line and the effect of stimulus alignment. Psychonomic Science, 1964, 1. 143-144.

SHEPARD, R., \& METZLER, J. Mental rotation of three-dimensional objects. Science, 1971, 171, 701-703.

SMITH, N. Matching ability as a factor in first grade reading. Journal of Educational Psychology, 1928, 19, 560-571.

SNODGRAss, J. Objective and subjective complexity measures for a new population of patterns. Perception \& Psychophysics, 1971, 10. $217-224$.

Staller, J., \& Sekuler, R. Mirror-image confusions in adults and children: A nonperceptual explanation. American Journal of Psychology, 1976, 89, 253-268.

WoLfF. P. Mirror-image confusability in adults. Journal of Experimental Psychology, 1971, 91, 268-272.

\section{NOTES}

1. There are actually four stimulus-response mapping criteria in Experiment 1: a left-right criterion (pairs 1 and 2 are "same," 3 and 4 are "different"); an up-down criterion (pairs 5 and 6 are "same," 7 and 8 are "different"); an in-out criterion which refers to horizontal pairs ( 1 and 2 are "different," 3 and 4 are "same"); and an in-out criterion which refers to vertical pairs (5 and 6 are "different," 7 and 8 are "same"). The important contrast is between Group 1 (which used left-right/up-down) vs. Group 2 (which used horizontal in-out/vertical in-out). For ease of explication in this paper, left-right will refer to Group 1, and in-out will refer to Group 2.

2. After the testing was completed, subjects were informally interviewed concerning their participation in the experiment. Three subjects in the in-out group revealed that they had not followed instructions. The data for these three subjects were therefore discarded. In addition, the data for one subject in the left-right group were discarded because of extremely slow response times. An additional four subjects were tested in the above conditions to make up the loss.

3. The complex stimuli were generated as follows. After coordinates for points on the six lines were determined for the simple patterns (Level 1) the points were dispersed about each line by a constrained, one-dimensional random walk. On each cycle of the walk, every point in a pattern was displaced with probability $=.5$. Points on vertical lines could be displaced with equal probability to the left or right; points on horizontal lines could be displaced with equal probability up or down. The size of each displacement $\left(2.5^{\prime}\right)$ was constant. The loci of the original 
"simple" lines constituted a nonabsorbing boundary beyond which a point could not be displaced. This can be illustrated by imagining for a moment that the pattern components have a fourth side. A point in the display could assume any value within this imaginary square but could not assume a value outside it.

The random walk method afforded a way to create a continuum of complexity while preserving the directional character of the patterns. Complexity (assumed to be a monotonic function of randomness) was based on the number of steps in the random walk. More steps produced greater dispersion, and therefore, more complex patterns. For the simple patterns (Level 1), zero steps were taken, producing no dispersion of points. To create the most complex patterns (Level 5), each point in the stimulus was subjected to an 80-step-long random walk, producing considerable dispersion. Levels 2-4 represent intermediate levels of complexity: 20-, 40-, and 60-step-long random walks, respectively.

4. This unusual trend term is produced from the second set of coefficients in Table 1.

(Received for publication May 6, 1977; revision accepted September 12, 1977.) 